\title{
Adaptive image enhancement algorithm based on the model of surface roughness detection system
}

\author{
Jie Tian and Xijie Yin ${ }^{*}$
}

\begin{abstract}
In view of the relatively high noise interference and halo phenomenon of the traditional adaptive image enhancement algorithm based on the unsharp masking method, a kind of adaptive image enhancement algorithm based on the integration of the model of surface roughness detection system (hereinafter referred to as MSRDS for short) is put forward in this paper. Through the design of the model of the surface roughness detection system, non-linear segmentation, denoising, and adaptive amplification are carried out on the details of the image under this system model. The dynamic range compressed image base layer and the adaptively enhanced image detail layer are non-linearly superimposed to obtain the final enhanced image. Finally, through the comparative experiment analysis, it demonstrates that the method put forward in this paper can suppress the interference noise and the halo phenomenon of the image very well while carrying out dynamic range compression and detail amplification of the adaptive image effectively. And the result thus obtained is very suitable for the back-end image processing of the actual thermal infrared imager.
\end{abstract}

Keywords: Adaptive image, Image enhancement, Surface roughness detection, Image processing

\section{Introduction}

With the development of industrial production technology, there is an increasing demand for product precision and surface roughness of parts. The traditional surface roughness measurement method is mainly compared with the sample block and compared with the measured surface according to vision or touch. According to different comparison methods, it is divided into comparative method, impression method, stylus method, interference method, and light-cut method. Nowadays, these traditional measurement methods are more and more unable to meet the demand of surface roughness detection of today's production, so continuing a one of a kind is a new way to solve this problem. The image detection method belongs to a non-destructive technology detection method. Since image processing and information processing can be used, more and more researchers are now studying how to use the image processing method to measure the surface roughness.

* Correspondence: yxjshandong@126.com

School of Data and Computer Science, Shandong Women's University, Jinan 250300, China

(c) The Author(s). 2018 Open Access This article is distributed under the terms of the Creative Commons Attribution 4.0 International License (http://creativecommons.org/licenses/by/4.0/), which permits unrestricted use, distribution, and reproduction in any medium, provided you give appropriate credit to the original author(s) and the source, provide a link to the Creative Commons license, and indicate if changes were made.
With the maturity of the surface roughness detection technology, all the thermal infrared imagers today have extremely high dynamic range (HDR), which can reach up to 14 bits or even higher (in this paper, 14 bits is adopted as an example) [1,2]. The relatively high dynamic range ensures that the thermal imager can still clearly distinguish the details of the temperature change in the scene where the temperature change is extremely great [3-5]. However, high dynamic range can cause a mismatching issue between the original image captured and the back-end device [6, 7]. As the traditional back-end display and processing devices are all based on 8-bit gray scale images, the dynamic range compression (DRC) of the adaptive image is a very important aspect in the back-end image processing of the thermal infrared imager [8-10]. The DRC processing technology has been greatly developed in recent years. Among them, the methods of automatic gain control (AGC) and histogram equalization (HE) have been most widely applied [11, 12]. The AGC method maps 14-bit raw data linearly to 8-bit pixel values after eliminating the interference noise $[13,14]$. On the other hand, the HE distributes the gray

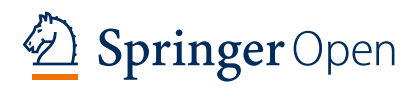


level of the probability density function (PDF) of the original image to more output gray levels, while merging or compressing the relatively small gray levels of the PDF to enhance the DRC of the image and the contrast. However, the HE method also has some obvious defects [15]. In view of the insufficiency of AGC and HE processing methods in the detailed control [16], some of the more complex algorithms have also been developed [16-18]. With the increasing application of computer information technology, computer graphics-related knowledge has been used in many fields, such as film production, games, and computer-intelligent image enhancement, where computer intelligent image enhancement technology has been applied. In most cases, the resolution of computer intelligent images is relatively high. Generally, the redundant information part needs be removed from the images to improve the efficiency and level of information processing. So far, many image enhancement algorithms are aimed at single, static computer-intelligent images, and there are relatively few studies on dynamically enhanced ones. Through the review of related literatures, it can be concluded that there are still many algorithms for image enhancement, which can be divided into vertex deletion, region merging, and repeated edge contraction specifically. Analysis shows that the most widely used is the edge contraction method. In such algorithm, an SAR framework is constructed based on the fusion of computer-intelligent images to achieve effective refinement of images on this basis. Most of the algorithms previously proposed were for static images, and the effects thus obtained were also very prominent. However, such algorithms fail to take the time consistency issue into full consideration. Hence, it is difficult to apply them directly to dynamic networks.

In view of the above deficiencies, an adaptive image enhancement algorithm based on the integration of the surface roughness detection system model is put forward in this paper. Through the construction of the surface roughness detection system model, the gray levels of input and output are defined under this model to implement the image segmentation, detail adaptive amplification, and dynamic range compression of the base layer part. In this process, the halo phenomenon is eliminated very well and the interference noise of the image details is suppressed effectively.

\section{Method-construction of surface roughness detection model}

Through analysis, it can be found that in order to solve this problem, it is necessary to make necessary improvements to the segmentation and superimposition processes of the two images. In the actual processing of the images, it is not necessary to follow the inherent addition and subtraction operations. And some non-linear operations can be designed in accordance with the actual needs based on the noise level of the actual images and some characteristics of the mask itself. In addition, in the design of the homomorphic filter, the image is first subjected to log transformation, that is, after the image data are transformed into the logarithmic domain, the post-processing of the image (such as the low-pass filtering) is carried out. The experiment shows that the effect after such processing is greatly improved compared with the traditional design methods. Inspired by this method, the image operation in the process is redesigned in this paper. And the basic idea is shown in Fig. 1.

In Fig. 1, $\Phi()$ stands for a function that can be customized. If $\Phi()$ is defined as $y=x$, the above system is not different from the normal operation, that is, the normal linear operation. However, in the actual design, $\Phi()$ is generally defined as the non-linear function. At this point, the entire operation block diagram will no longer maintain the normal linear operation properties. Therefore, it can be referred to as the generalized linear operation. Hence, the operation is redefined for this purpose. It is assumed that add,sub, and mult stand for the addition operation, the subtract operation, and the multiplication operation, which is represented by the following formula, respectively:

$$
\begin{aligned}
& x_{1} \text { add } x_{2}=\Phi^{-1}\left[\Phi\left(x_{1}\right)+\Phi\left(x_{2}\right)\right], \\
& x_{1} \text { subx } x_{2}=\Phi^{-1}\left[\Phi\left(x_{1}\right)-\Phi\left(x_{2}\right)\right], \\
& {\text { amult } x_{1}}=\Phi^{-1}\left[a \times \Phi\left(x_{1}\right)\right],
\end{aligned}
$$

In the above three equations, $x_{1}$ and $x_{2}$ stand for two-channel input signals, and the signals may be one-dimensional or multi-dimensional arrays; $\alpha$ stands for a real number and $\Phi()$ defines a non-linear function in accordance with actual needs. In view of the different requirements of the image enhancement, the following $\Phi()$ functions are designed, respectively:

$$
\begin{aligned}
& \Phi(x)=\frac{R_{\text {after }}}{2} \times\left(\sqrt{\frac{x}{R_{\text {before }}-x}}-\sqrt{\frac{x}{R_{\text {before }}-x}}\right), \\
& \Phi^{-1}(x)=\frac{R_{\text {before }}}{2} \times\left(\frac{x}{\sqrt{R_{\text {after }}^{2}+X^{2}}}+1\right),
\end{aligned}
$$

in which $R_{\text {before }}$ stands for the gray level of the input image and $R_{\text {after }}$ stands for the gray level after the

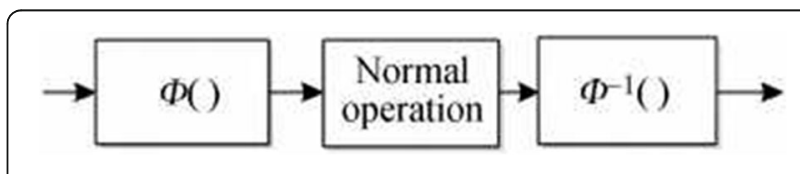

Fig. 1 Block diagram of surface roughness detection 
operation is completed. In most cases, $R_{\text {before }}$ is equal to $R_{\text {after }}$. However, when some of the calculations involve the dynamic range compression of the image, $R_{\text {before }}$ is not equal to $R_{\text {after }}$. In addition, to facilitate the understanding, two variables, $R_{\text {before }}$ and $R_{\text {after }}$ are extracted outside the operation. And a model of the original surface roughness detection is established, as shown in Fig. 2.

After the construction of the surface roughness detection, Eqs. (4) and (5) are converted to the following:

$$
\begin{aligned}
& \Phi(x)=\frac{1}{2} \times\left(\sqrt{\frac{x}{1-x}}-\sqrt{\frac{1-x}{x}}\right), \\
& \Phi^{-1}(x)=\frac{1}{2} \times\left(\frac{x}{\sqrt{1+x^{2}}}+1\right),
\end{aligned}
$$

Equations (6) and (7) are introduced into the Eqs. (1), (2), and (3) to obtain the following:

$$
\begin{aligned}
x_{1} \text { add } x_{2}= & \frac{1}{2} \\
& \times\left(\frac{\Phi\left(x_{1}\right)+\Phi\left(x_{2}\right)}{\sqrt{1+\left(\Phi\left(x_{1}\right)+\Phi\left(x_{2}\right)\right)^{2}}}+1\right), \\
x_{1} \text { subx }_{2}= & \frac{1}{2} \times\left(\frac{\Phi\left(x_{1}\right)-\Phi\left(x_{2}\right)}{\sqrt{1+\left(\Phi\left(x_{1}\right)-\Phi\left(x_{2}\right)\right)^{2}}}+1\right), \\
x_{1} \text { mult }_{2}= & \frac{1}{2} \times\left(\frac{\alpha \times \Phi\left(x_{1}\right)}{\sqrt{1+\left(\alpha \times \Phi\left(x_{1}\right)\right)^{2}}}+1\right),
\end{aligned}
$$

\section{Adaptive image enhancement algorithm}

In this paper, a kind of new adaptive image enhancement algorithm is put forward. And the specific process design is shown in Fig. 3.

The system first extracts the base layer image of the image through bilateral filtering and then makes use of the model of surface roughness detection system (MSRDS) operation put forward in Section 3 to segment the details of the image from the original image. Subsequently, the dynamic range of the base layer is compressed

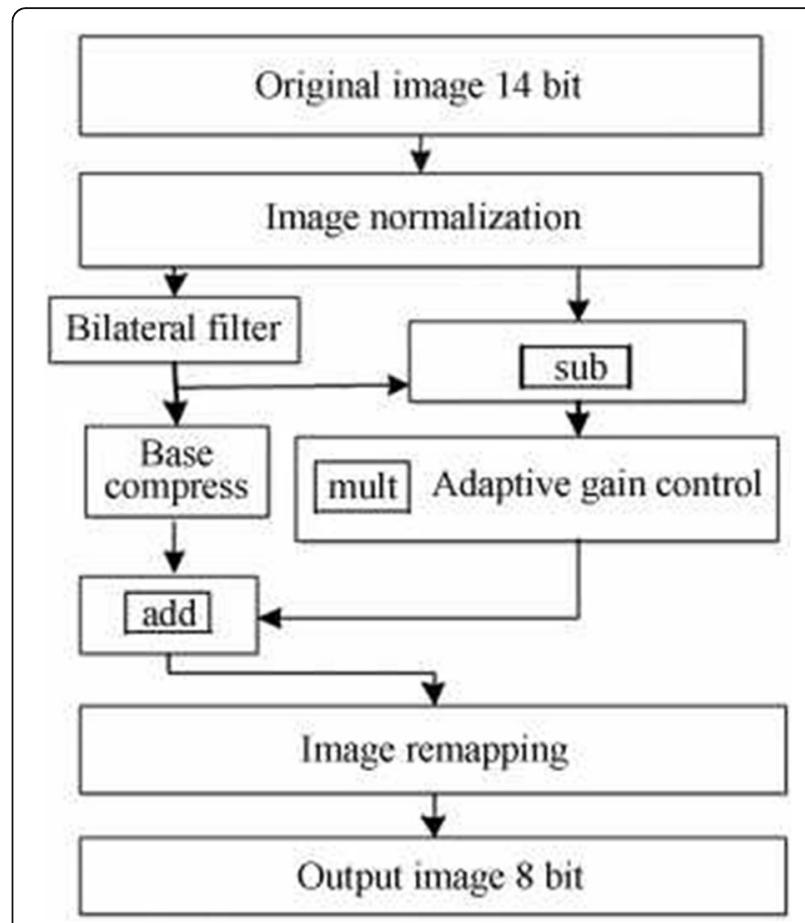

Fig. 3 Block diagram of the surface roughness detection system

and the detail layer is enhanced and amplified. Among them, MSRDS multiplication operation can be used to implement DRC at the base layer, and the adaptive incremental control is adopted at the detail layer to enlarge detail information. Finally, the detail layer and the base layer images are added together by the MSRDS addition operation to obtain an image after the enhancement.

In the traditional adaptive image enhancement algorithm, no matter the subtraction algorithm or the template matching algorithm is adopted, it is necessary to carry out one-to-one match and calculation on the pixels of the template image and the image to be tested. The error caused by the image offset is a problem that cannot be eradicated. The solution can only be obtained by increasing the image registration and correction algorithm, which will result in the impact on the real-time performance of the algorithm. In the residue detection algorithm based on the fusion of the Moore nearest neighbor model, the residue is highlighted, which has effectively avoided the image offset problem. At the same time, the vector machine classification is introduced,

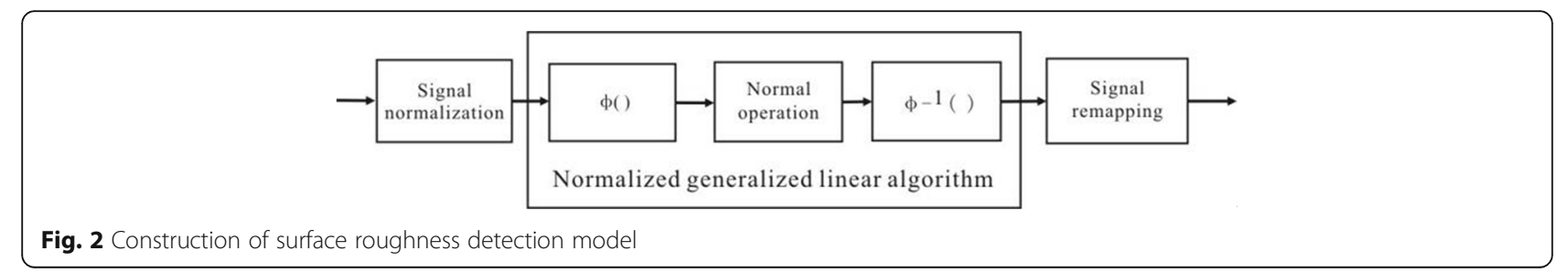


which has reduced the misjudgment caused by the image offset and improved the detection accuracy.

In the new image enhancement algorithm, three stages are required in general: the core algorithm design, the experimental design and implementation, and the experiment. This is a cyclic process of optimization and improvement, in which multiple aspects of work need to be completed.

Definition 1 Core algorithm development researchers are most interested in the modeling of the image enhancement algorithm and the implementation of its basic programming, which is also referred to as the new algorithm or target algorithm, the "Useful work" $W_{\text {useful }}$.

Definition 2 Auxiliary development is the basic image algorithm research and development to support the core algorithm development, as well as the GUI visualization development for human-computer interaction, which is "Extra work" $W_{\text {extra }}$.

Therefore, the development efficiency is $Z=\frac{W_{\text {有 }}}{W_{\text {有 }}+W_{\text {额 }}}$, in which $W_{\text {useful }}$ is difficult to change in general, and it is preferable that the algorithm and interface resource reuse and inheritance mechanisms can be proved in the development environment to reduce or avoid the $W_{\text {extra }}$ and improve the efficiency.

$$
\begin{aligned}
& W_{\text {有 }} \\
& W_{\text {useful }} \\
& W_{\text {额 }} \\
& W_{\text {extra }}
\end{aligned}
$$

The model put forward in this paper includes the processing mode and the operating mode.

The visual image enhancement mode flow and the specific steps are as follows.

Step 1: Acquire the original image data

Step 2: Carry out gray scale processing on the original image

The specific process of the gray scale algorithm is as follows: Obtain the R, G, and B components of each pixel; calculate the R, G, and B components of each pixel in accordance with Eq. (11); calculate and obtain the temp overlay on the original pixel; and repeat the aforementioned process from the first pixel to the last pixel in continuous loop.

$$
\text { temp }=(r * 299+g * 587+b * 114+500) / 1000 .
$$

Step 3: Carry out median filtering on the gray scale image
The specific process of the median filtering algorithm is as follows: Obtain the gray scale value of nine pixels through a $3 \times 3$ sliding window; calculate the gray scale value of nine pixels in accordance with Eq. (12); traverse the whole image in turn by the $W$ template; and replace the pixel value at the center point of the template with the median value obtained in the area covered by the template with the size of $W$.

$$
g(x, y)=\operatorname{med}\{f(x-k, y-l),(k, l \in W)\},
$$

in which med stands for the median operation, that is, the pixels in the $W$ template are first sorted by size, and then, the median value of the pixels in the $W$ matrix is obtained. $f(x, y)$ and $g(x, y)$ stand for the original image and the processed image, respectively.

Step 4: Perform three-layer adaptive decomposition on the filtered gray image.

Adaptive transform is the basis of adaptive decomposition of images, and the adaptive basis function is as follows:

$$
\Psi_{a, \tau}(t)=\frac{1}{\sqrt{a}} \Psi\left(\frac{t-\tau}{a}\right) a, \tau \in R ; a>0,
$$

in which $a$ stands for the scaling factor and $\tau$ stands for the translation factor. Let $\Psi(x, y)$ stand for the twodimensional basic adaptation, then the two-dimensional basic adaptation is defined as follows:

$$
\begin{aligned}
W T_{f}\left(a ; b_{1}, b_{2}\right) & =\left\langle f(x, y), \Psi_{a ; b_{1} ; b_{2}}(x, y)\right\rangle \\
& =\frac{1}{a} \iint f(x, y) \Psi^{*}\left(\frac{x-b_{1}}{a}, \frac{y-b_{2}}{a}\right) d x, d y,
\end{aligned}
$$

in which $\Psi_{a ; b_{1} ; b_{2}}(x, y)=\frac{1}{a} \iint f(x, y) \Psi^{*}\left(\frac{x-b_{1}}{a}, \frac{y-b_{2}}{a}\right)$ stands for the scale expansion and two-dimensional displacement of $\Psi(x, y)$. $\frac{1}{a}$ stands for the normalization factor introduced to ensure that the energy remains unchanged before and after the adaptation.

After the image is adaptively decomposed, the low-frequency coefficient, horizontal high-frequency coefficient, vertical high-frequency coefficient, and oblique high-frequency coefficient of the image can be obtained.

Step 5: In accordance with Eq. (5), low-frequency decomposition coefficient of the image after a three-layer adaptation is enhanced. The highfrequency decomposition coefficient is attenuated to achieve the image enhancement effect, that is, the adaptive passivation. 


$$
\left\{\begin{array}{l}
c(i)=4 * c(i) ; c(i)>405 \\
c(i)=0.1 * c(i) ; c(i) \leq 405
\end{array}\right.
$$

in which $c(i)$ stands for the coefficient after two-dimensional decomposition is carried out on the image.

Step 6: Enhance the image after the Moore nearest neighbor model is applied. The specific calculation in accordance with Eq. (16) is as follows:

$$
g(x, y)=\left\{\begin{array}{l}
0, f(x, y)<\text { threshold } \\
1, f(x, y)>\text { threshold }
\end{array}\right.
$$

in which $f(x, y)$ stands for the image after the adaptive decomposition and reconstruction, $g(x, y)$ stands for the image after the enhancement, and threshold stands for the threshold value selected for the enhancement.

Step 7: Perform statistical calculation on the number of pixels 0 and 1 for the enhanced image and store the statistical data as an array, such as:image $[i]=\{$ pixel $\underline{0}$ nums, pixel 1 nums $\}$.

Step 8: For cycle steps $1 \sim 7$ of multiple images in the same mold cavity, the array of each image is obtained and input into the support vector machine. The property of the arrays with residue is marked as 1 , and those without residue marked as -1 to obtain the specific method of the two-class classification mathematical judgment model for determining whether there is a residue as follows:

Select the linear kernel function $K\left(x_{i}, x_{j}\right)=x_{i}, x_{j}$ to obtain two types of $\operatorname{samples}\left(x_{i}, x_{j}\right), i=1,2, \ldots, n$, in which $n$ stands for the number of samples thus obtained. When $x_{i}$ falls under sample class $\omega_{1}$ that does not contain residues, $y_{i}=1$ and when $x_{i}$ falls under sample class $\omega_{2}$ that does not contain residues, $y_{i}=-1$, the support vector machine is used for processing to obtain the classification model as follows:

$$
f(x)=\operatorname{sgn}\left\{\sum_{i=1}^{n} a_{i}^{*} y_{i}\left(x_{i} \cdot x\right)+b^{*}\right\} \quad \text { parameters } a^{\prime \prime} \text { and }
$$
$b^{*}$.

The operating process of the adaptive image enhancement algorithm and the specific steps are as follows.

Step 1: Obtain the judgment image data

Step 2: Perform gray scale processing on the judgment image

Step 3: Carry out filtering on the gray scale image

Step 4: Perform three-layer adaptation on the filtered gray scale image

Step 5: Apply the Moore nearest neighbor model on the three-layer adaptive image to highlight the contour of the residue
Step 6: Enhance the image after the Moore nearest neighbor model is applied

Step 7: Perform statistical calculation on the number of pixel 0 and 1 for the enhanced image and store the statistical data as an array

Step 8: Adopt the two-class classification mathematical model to judge whether there is residue for the array in Step 7, to determine whether the array is an array that contains the residue image

\subsection{Image construction and remapping}

As the pixel data of the input image is represented by 14 bits, the possible distribution of the actual image pixel gradation is $0 \sim 16383$. Therefore, when the image construction processing in Fig. 3 is carried out, it can be simply obtained through the image divided by $R_{\text {before }}\left(R_{\text {be- }}\right.$ fore $=16383)$. However, from the imaging characteristics of the adaptive images, it can be seen that the images collected by the infrared detectors are often distributed in one of the regional segments from 0 to $16383 \mathrm{seg}$ ments instead of being distributed in the entire gray scale space. In order to obtain better results for the subsequent processing, the selection of $R_{\text {before }}$ during the construction of the image does not have to be constrained by the theoretical gray scale distribution of the image. On the contrary, an ideal image gray scale distribution can be re-determined after the influence of the noise is eliminated. In addition, in order to ensure that the above proposed algorithm can be applied in the most effective manner, the gray scale with the maximum probability density function (PDF) on the gray scale histogram of the image is mapped to the zero value of the MSRDS (that is, $1 / 2$ ). And $R_{\text {before }}$ can be determined by using the probability density function (PDF) and the cumulative probability distribution function (CDF) of the image gray scale with the formula is as follows:

$$
\begin{aligned}
& r_{\min }=\min \left\{r \mid C D F(r)>P_{\text {noise }} / 2\right\} \\
& r_{\min }=\max \left\{r \mid C D F(r)<1-P_{\text {noise }} / 2\right\} \\
& r_{1 / 2}=r \mid P D F(r)>P D F(r+\delta) \\
& R_{\text {before }}=2 \times \max \left\{r_{\max }-r_{1 / 2}, r_{1 / 2}-r_{\min }\right\}
\end{aligned}
$$

in which $P_{\text {noise }}$ stands for the level of the camera, which is described in the detector. It can also be selected through the $R_{\text {after }}$ measured in the experiment in a simple manner in accordance with the requirements for the subsequent display equipment, which is selected as $R_{\text {after }}=255$ in general.

\subsection{Dynamic range compression at the image base layer} After the original image has been detected through the surface roughness, the base layer of the image is obtained. From the above analysis, it can be known that the settings of the $\delta$ and $\delta$ in the filter are very critical. It 
is set that $\delta_{s}=\max$ (Width, Height) $/ 16$, and $\delta_{g}$ is set to about $10 \%$ of the dynamic range of the image. The intrinsic meaning of these two parameter settings is as follows: If the pixel area in the image area is smaller than approximately $10 \% \delta_{g}$ left of the total gray scale range, it can be regarded as the detail change of the image. The $\delta_{s}$ set indicates that the MSRDS filter scope is $1 / 16$ the width of the image. The pixels beyond this range have very little effect on the filtering results, which tend to be almost zero and are negligible.

After the base layer image is obtained, it is necessary to carry out dynamic range compression. From Fig. 4, it can be seen that in the MSRDS operation, if the signal is multiplied by the last number that is less than 1 , the value near the $G L-\Phi$ zero value of the signal will be compressed. On the basis of this principle, the base layer image is multiplied with $\alpha$ in this paper to achieve dynamic range compression. In this experiment, $\alpha=0.2$, and the results are shown in Fig. 4. From Fig. 4, it can be seen that after the processing of the MSRDS multiplication, the dynamic range of the base layer image is compressed. However, as the base layer of the image only summarizes the approximate gray scale distribution of the image, the detailed information of the image is relatively missing, which requires the image at the detail layer to supplement.

\subsection{Acquisition of image detail layer}

By subtracting the base layer image obtained by 4.2 from the original image, the detail layer of the image can be obtained. The subtraction operation of the image can adopt either the traditional subtraction method or the MSRDS put forward in Section 3. The MSRDS effect diagram can be obtained from Eq. (21) and Fig. 2. And the results are shown in Fig. 5 as follows:

In accordance with the analysis equation $y(\delta)=(x+\delta$ ) subx in Fig. 5, $x$ is regarded as a constant and $\delta$ is regarded as a variable. And the following conclusions can be drawn:

$$
\left.\frac{d y}{d \delta} \rightarrow 0\right|_{\delta \rightarrow 1, x \rightarrow 0} ;\left.\frac{d y}{d \delta} \rightarrow \infty\right|_{\delta \rightarrow 0, x \rightarrow 0} ;
$$

From Eq. (12) and Fig. 5, it can be seen that when the two subtraction numbers are very close (that is, $\delta \rightarrow 0$ ), the MSRDS operation has certain amplification effect on the difference between the two numbers. At this point, it is highly sensitive to the change of 8 ; while when the two subtraction numbers are significantly different (that is, $\delta \rightarrow 1$ ), the MSRDS has the compression effect on the difference of the two numbers, and it will be less sensitive to the change of 8 . Through the comprehensive analysis, it can be known that the MSRDS can extend small differences while compress large differences.

\section{Experiment and result discussions}

In order to verify the experimental results of the algorithm model put forward in this paper, the proposed algorithm is compared with the commonly used AGC algorithm, the histogram equalization (HE), and the proposed MSRDS algorithm. The AGC and HE are selected because they are commonly used and effective methods to implement the image enhancement, while the MSRDS is selected because it adopts the same MSRDS as the edge preserving filter and is the same as that in the method put forward in this paper. And the parameters of the MSRDS algorithm are set in accordance with the default value. In addition, the selected scenes are three highly representative ones: (1) The scene with relatively long outdoor distance (approximately $2 \mathrm{~km}$ away), (2) the scene with relatively close outdoor distance (within $100 \mathrm{~m}$ ), and (3) the indoor experimenter. The results of the three groups of experiments are shown in Figs. 6 and 7.

It can be seen from Fig. 6 that the AGC method has relatively smooth image surface, low contrast, and blurred image. And when the HE model and MSRDS deal with the distant scenes outdoors, although the images have been locally enhanced, it also results in the blurring in some local areas (arrow B and arrow C). However, when local area is enhanced, the method put forward in this paper can still display the details outside

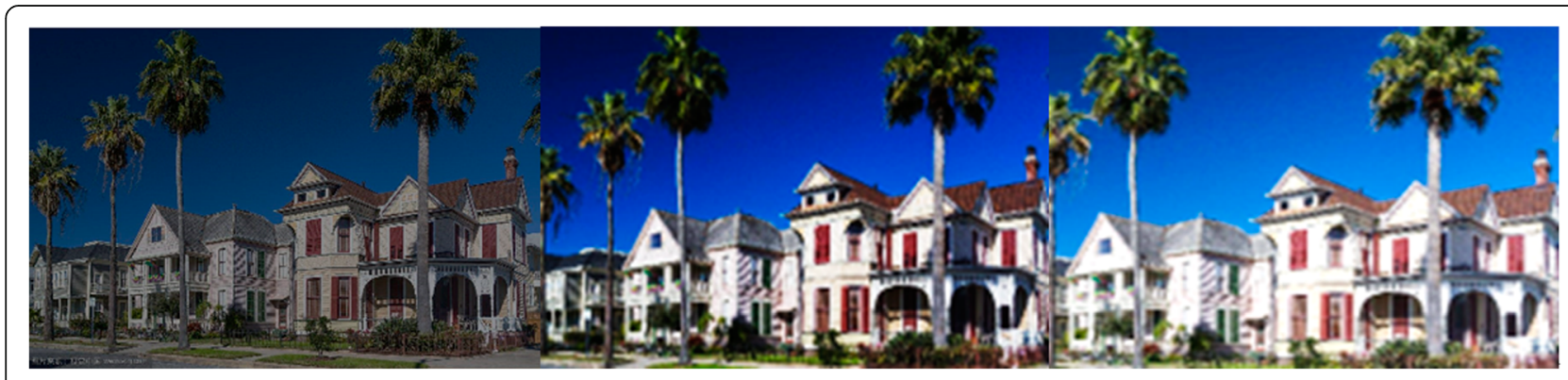

Fig. 4 Image base layer processing 


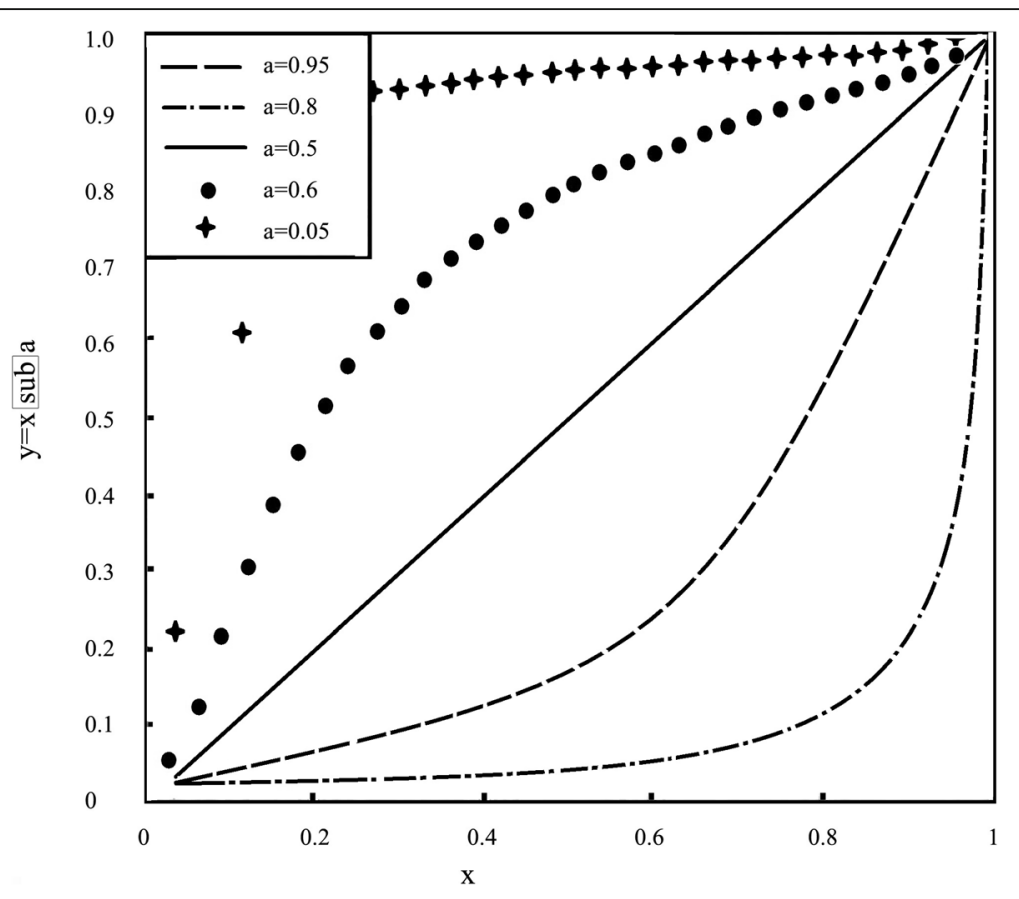

Fig. 5 MSRDS subtraction

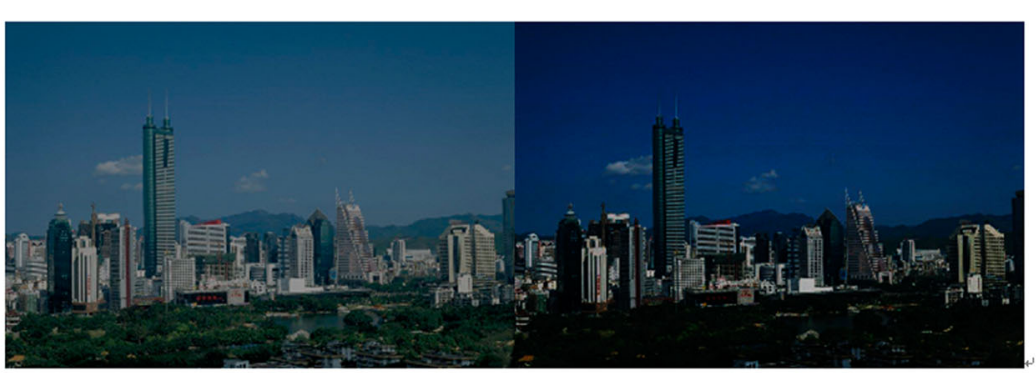

(a) Result of AGC algorithm (b) Result -of MSRDS model algorithm

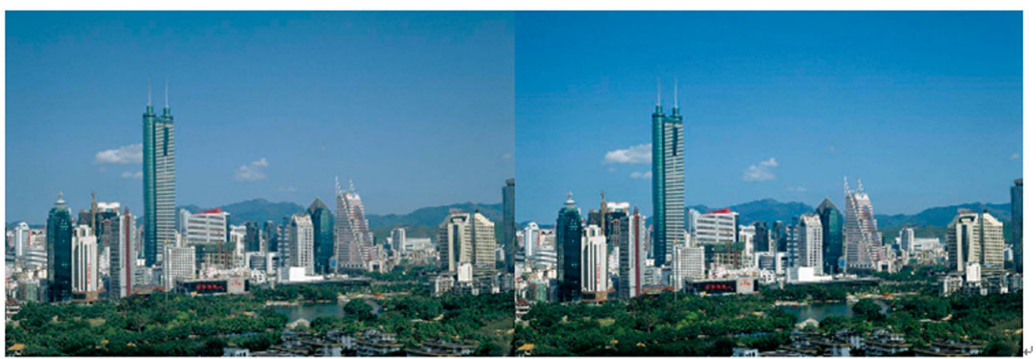

(c) Result of HE algorithm (d) Result of the method proposed this paper

Fig. $\mathbf{6}$ a-d Comparison of the algorithms for outdoor long-distance scenes 


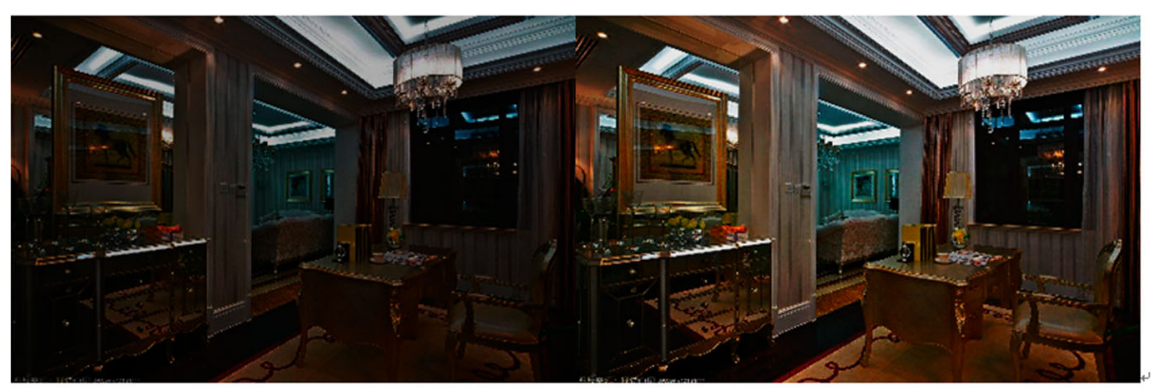

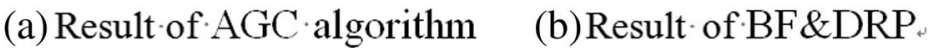

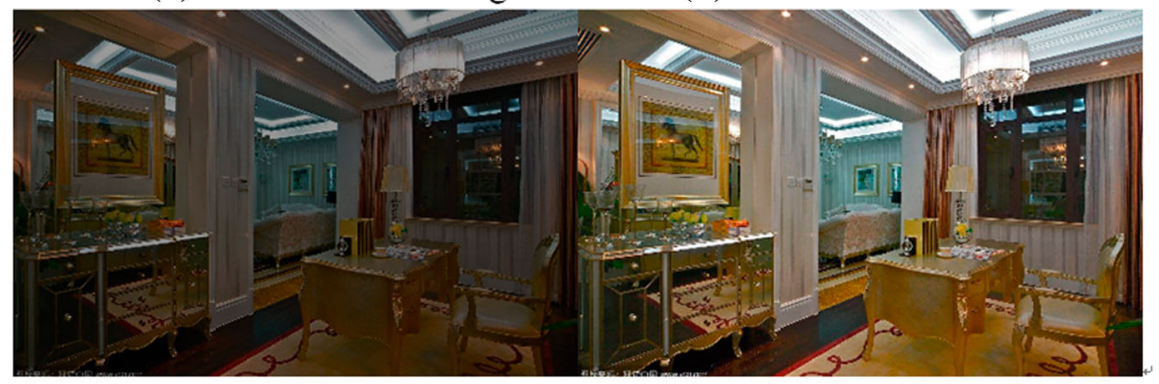

(c) Result of HE …… (d) Result of the method proposed this paper

Fig. 7 a-d Comparison of the processing results of several algorithms for the indoor scenes

$2 \mathrm{~km}$ (crane wire outside $2 \mathrm{~km}$, that is, arrow D). Among them, the processing result of MSRDS has relatively high contrast, but the whole image is excessively sharp and the overall effect of the image is insufficient. During the programming of the MSRDS algorithm, the default values of the original literature are adopted at this point. The selection of the parameters still needs to be optimized. If the parameters can be adjusted to the optimal ones, better results can be obtained.

Figure 7 is an external field image which is relatively close to the observer. From the visual effect of the image, the AGC algorithm and the HE algorithm are significantly blurred at the arrow $\mathrm{A}$ and arrow $\mathrm{C}$, while the $\mathrm{BF}$ and DRP algorithm shows excessively sharpened halo phenomenon at the arrow B. In particular, the halo phenomenon at the upstairs windows of the residents is very serious. The processing results of the method put forward in this paper have relatively good overall effect. For example, the house structure streak at the arrow D is still clearly visible.

\section{Conclusions}

In this paper, a new adaptive image enhancement algorithm technique is designed through the analysis of the imaging characteristics of the adaptive images. Firstly, the base layer part of the image is segmented by MSRDS. Then, the MSRDS operation is carried out to segment the base layer, and the detail image is enlarged to achieve the dynamic compression of the base layer. In the MSRDS operation definition, the data overflow problem is solved effectively, which has eliminated the halo phenomenon of the image and suppressed the image noise appropriately. The comparison experiments show that the method put forward in this paper has significant improvement in the overall performance of the image, the detail performance, and the mean contrast enhancement of the image, which is a very important reference value for the actual image processing in the back-end of the infrared imager.

The method put forward in this paper has a clear idea and is suitable for a hardware design. The algorithm proposed in this paper has been successfully implemented in the DE2-115 FPGA development version, which is not described in this paper due to the limited space. However, in the design of this paper, due to the large number of parameters involved, whether the settings of these parameters are appropriate or not will have relatively great impact on the results in general. In the subsequent work, how to adjust these parameters adaptively will be a major research direction within the subject.

\section{Abbreviations}

AGC: Automatic gain control; DRC: Dynamic range compression; HDR: High dynamic range; HE: Histogram equalization; MSRDS: Model of surface roughness detection system; PDF: Probability density function 


\section{Acknowledgements}

The authors thank the editor and anonymous reviewers for their helpful comments and valuable suggestions.

\section{Availability of supporting data}

We can provide the data.

\section{Author details}

Jie Tian was born in Shandong, China, in 1983. She received the B.Sc. degree in Computer science and technology from Shandong Agricultural University, Taian, China, and M.Sc. degree in Computer software and theory from Taiyuan University of Science and Technology, Taiyuan, China, and her current research interests include Intelligent computing, machine learning, and image processing.

Xijie Yin was born in Shandong, China, in 1965. She received the Master degree from Shandong University, China. Now, she works in the School of Data and Computer Science, Shandong Women's University, Her research interests include cloud security and image processing.

\section{Funding}

This work was supported in part by high level research project Cultivation Fund of Shangdong women's university, soft science project of Shandong (Grant No. 2014RKB01647) and data mining research innovation team of Shandong Women's University, China.

\section{Authors' contributions}

All authors take part in the discussion of the work described in this paper. JT wrote the first version of the paper and did a part in the experiments of the paper. $X Y$ revised the paper in a different version. Both authors read and approved the final manuscript.

\section{Competing interests}

The authors declare that they have no competing interests.

\section{Publisher's Note}

Springer Nature remains neutral with regard to jurisdictional claims in published maps and institutional affiliations.

Received: 8 July 2018 Accepted: 20 September 2018

Published online: 11 October 2018

\section{References}

1. B. Li, S. Wang, J. Zheng, L. Zheng, Single image haze removal using content-adaptive dark channel and post enhancement. IET Comput. Vis. 8(2), 131-140 (2014)

2. B. Li, W. Xie, Adaptive fractional differential approach and its application to medical image enhancement. Computers \& Electrical Engineering 45, 324 335 (2015).

3. J.R. Tang, N.A.M. Isa, Adaptive image enhancement based on bi-histogram equalization with a clipping limit. Computers \& Electrical Engineering 40(8), 86-103 (2014)

4. W. Zhao, Z. Xu, J. Zhao, F. Zhao, X. Han, Variational infrared image enhancement based on adaptive dual-threshold gradient field equalization. Infrared Phys. Technol. 66(66), 152-159 (2014).

5. Z. Zhou, S. Sorensen, H. Zeng, M. Hawrylycz, H. Peng, Adaptive image enhancement for tracing $3 \mathrm{~d}$ morphologies of neurons and brain vasculatures. Neuroinformatics 13(2), 153-166 (2015).

6. J.W. Wang, N.T. Le, J.S. Lee, C.C. Wang, Color face image enhancement using adaptive singular value decomposition in Fourier domain for face recognition. Pattern Recogn. 57(C), 31-49 (2016).

7. L.T. Yuan, S.K. Swee, T.C. Ping, Infrared image enhancement using adaptive trilateral contrast enhancement. Pattern Recogn. Lett. 54, 103-108 (2015).

8. Z. Ling, Y. Liang, Y. Wang, H. Shen, Adaptive extended piecewise histogram equalisation for dark image enhancement. IET Image Process. 9(11), 10121019 (2015).

9. S. Dai, Q. Liu, P. Li, J. Liu, H. Xiang, Study on infrared image detail enhancement algorithm based on adaptive lateral inhibition network. Infrared Phys. Technol. 68, 10-14 (2015).

10. H. Gupta, K.K. Sharma, S.D. Joshi, Resource efficient FPGA implementation of adaptive image enhancement algorithms. IETE J. Res. 61(4), 429-439 (2015).
11. H. Wang, To, S, C.Y. Chan, Investigation on the influence of tool-tip vibration on surface roughness and its representative measurement in ultra-precision diamond turning. Int J Mach Tool Manu 69(3), 20-29 (2013).

12. T.D.L. Irvinefynn, E. Sanzablanedo, N. Rutter, M.W. Smith, J.H. Chandler, Measuring glacier surface roughness using plot-scale, close-range digital photogrammetry. J. Glaciol. 60(223), 957-969 (2014).

13. A. Krzeminski, S. Wohlhüter, P. Heyer, J. Utz, J. Hinrichs, Measurement of lubricating properties in a tribosystem with different surface roughness. Int. Dairy J. 26(1), 23-30 (2012).

14. M. Launhardt, A. Wörz, A. Loderer, T. Laumer, D. Drummer, T. Hausotte, Detecting surface roughness on SLS parts with various measuring techniques. Polym. Test. 53, 217-226 (2016).

15. Z. Liu, Q. Zhong, X. Yu, Q.J. Wang, Y. Zhang, Surface roughness measurement by depolarization method. Appl. Opt. 54(18), 56865690 (2015).

16. B. Kim, J. Seo, Measurement of surface roughness of plasma-deposited films using laser speckles. Appl. Surf. Sci. 359, 204-208 (2015).

17. P.B. Nagy, J.H. Rose, Surface roughness and the ultrasonic detection of subsurface scatterers. J. Appl. Phys. 73(2), 566-580 (1993).

18. G. Galante, M. Piacentini, V.F. Ruisi, Surface roughness detection by tool image processing. Wear 148(2), 211-220 (1991).

\section{Submit your manuscript to a SpringerOpen ${ }^{\circ}$ journal and benefit from:}

- Convenient online submission

- Rigorous peer review

- Open access: articles freely available online

High visibility within the field

Retaining the copyright to your article

Submit your next manuscript at $\boldsymbol{\nabla}$ springeropen.com 